\title{
Los exiliados argentinos en Venezuela ante el inicio de la transición a la democracia en La Argentina*
}

\author{
Mario Ayala**
}

La etapa final de la última dictadura militar y el inicio de la larga transición a la democracia en la Argentina a partir de fines de 1983 puso a los exiliados argentinos en Venezuela ante una serie de evaluaciones y decisiones políticas, personales y grupales complejas donde el retorno fue una posibilidad entre otras. El trabajo se apoya testimonios publicados y 32 entrevistas realizadas entre 2006 y 2011 con la metodología de la historia oral los cuales son analizados con un enfoque cualitativo. El análisis muestra que la mayoría de los retornos se realizaron entre los años1983 y 1985, en un contexto

\section{Introducción}

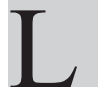

a experiencia del exilio es vivida y recordada en su doble condición: por sus facetas de miedo, dolor, soledad e incertidumbre permanente, pero también como espacio de descubrimiento, extrañamiento, reconocimiento $\mathrm{y}$

\footnotetext{
*Una versión previa de este texto fue presentada en la Mesa "Exiliados y retornados en los procesos de transición a la democracia en el Cono Sur: nuevas aproximaciones", VI Seminario Internacional Políticas de la Memoria: 30 años de democracia en Argentina. Logros y desafios, Centro Cultural de la Memoria Haroldo Conti, Buenos Aires, 7, 8 y 9 de noviembre de 2013.

*** Programa de Historia Oral, Instituto Interdisciplinario de Estudios e Investigaciones sobre América Latina de la Facultad de Filosofía y Letras de la Universidad de Buenos Aires. marioayala@filo.uba.ar
} 
muy poco favorable en términos económico y político y sin apoyo gubernamental, y que luego se espaciaron en un proceso que llega hasta el presente. Pero también se presentan casos de exiliados que en 1983 decidieron no retornar, otros que simplemente se fueron quedando y un grupo que fue forzado a permanecer fuera del país debido a procesos judiciales en su contra iniciados durante el periodo de terrorismo estatal y también en la década del ochenta, los cuales prescribieron recién avanzada la década de 1990, lo cual plantea el tema de la continuidad del exilio bajo regímenes democráticos.

Palabras claves: exiliados-argentinos-Venezuela-retorno-apertura democrática

The final stage of the military dictatorship and the beginning of the long transition to democracy in Argentina beginning in late 1983 put the Argentine exiles in Venezuela to a series of complex assessments and policies, personal and group decisions where the return was one possibility among others. Work published 32 testimonies and interviews conducted between 2006 and 2011 with the methodology of oral history which are analyzed with a qualitative approach supported. The analysis shows that most of the returns were made between 1985 and años1983 in a very unfavorable economic and political context in terms without government support, and then were spaced in a process that leads to the present. But cases of exiles who in 1983 decided not to return are also presented, others simply were left and a group that was forced to stay out of the country because of judicial proceedings against them initiated during the period of state terrorism and in the decade eighty, which advanced the newly prescribed 1990 s, which raises the issue of continuity of exile under democratic regimes.

Keywords: Argentina-Venezuela-exile-return-democratic opening

aprendizajes ${ }^{1}$. Al mismo tiempo esta condición exiliar implica casi siempre un deseo de retorno al país de donde se fue expulsado forzadamente. Sin embargo, este regreso no siempre es concretado debido a diferentes condicionantes y motivos de índole personal y político-social. Cuando los exiliados logran retornar, la sociedad que encuentran generalmente ya no es la misma en la que vivieron y a la cual evocaron durante el tiempo del destierro, que según las características del grupo y/o comunidad pudo durar desde algunos años hasta décadas. Comienza entonces un proceso complejo y abierto de intento de inserción-adaptación que puede resultar en la instalación y reintegración del exiliado en la sociedad expulsora, o en su retorno a la sociedad de acogida (o emigración a terceros paises). En esta perspectiva, retornar y des-exiliarse también son experiencias vividas

\footnotetext{
${ }^{1}$ SANHUEZA, Carlos, PINEDO, Javier (Eds.). El exílio latinoamericano, uma historia permanente. Em: SANHUEZA, Carlos, PINEDO, Javier. La pátria interrumpida: latinoamericanos em el exílio.Siglos XVIII-XX. 1ª Ed. Santiago: LOM Ed., 2010, p. 17.
} 
y recordadas en una doble condición: por lado, el exiliado vive un proceso de desestructuración e incertidumbre ante el enfrentamiento con la nueva realidad de su sociedad, el cual a veces implica un "nuevo exilio", revivir situaciones traumáticas, miedo a volver a ser represaliado y el desarraigo de la estabilidad construida en el destierro, y por el otro, las expectativas positivas en función del reencuentro con todo lo que fue obligado a dejar en el país: su familia, compañeros, amigos, lugares en común, trabajo, proyectos colectivos, familiares y personales ${ }^{2}$.

Estas disyuntivas, conflictos y sentimientos de los procesos de retorno y desexilio también fueron vividos por los exiliados argentinos en Venezuela durante el período de Terrorismo de Estado (1973-1983), quienes comenzaron a percibir el final de su destierro después la derrota de la dictadura en la guerra de las Islas Malvinas (abril-mayo de 1982) y, más tarde, con la posterior reactivación partidaria y sindical ante la crisis política y económica del gobierno militar, que culminó en el llamado a elecciones generales para octubre de 1983 y la elección de Raúl Alfonsín (Unión Cívica Radical, UCR) como presidente constitucional por el periodo 19831989. Así, la etapa final de la última dictadura militar y el inicio de la larga transición a la democracia en la Argentina puso a estos grupos de exiliados y sus familias ante una serie de evaluaciones y decisiones complejas acerca de regresar, o no, al país, que se decidieron mayormente en el ámbito personal-familiar, y a partir de las cuales activaron diversas estrategias, que no estuvieron exentas de conflictos, dudas, dilemas y miedos de abandonar la estabilidad lograda y recomenzar en un país signado por las crisis económica, la inestabilidad política, la continuidad del aparato represivo y una sociedad y un gobierno democrático que estigmatizaba y criminalizaba a la militancia política de izquierda (mayoritaria en el último exilio argentino) en el marco de la teoría de los "dos demonios" (los militares y

\footnotetext{
${ }^{2}$ FERNÁNDEZ, Jorge Christian. ¿Volver o no volver? Expectativas incertidumbres, dilemas y fracturasmfrente al desexilio entre los argentinos em Rio Grande do Sul, Brasil, em la década del ochebta. Ponencia en VI Seminario Internacional Políticas de la Memoria: 30 años de democracia en Argentina. Logros y desafios. Centro Cultural de la Memoria Haroldo Conti, Buenos Aires, 7, 8 y 9 de noviembr de 2013, p. 11.
} 
las guerrillas). Además, el regreso al país se tornó aún más difícil por no existir una política estatal coherente y activa de apoyo a la reinserción de los exiliados retornados por parte del gobierno nacional, por ejemplo en contraste con los casos de Uruguay o Chile $^{3}$. Al mismo tiempo, el gobierno argentino tampoco tuvo una política en otros temas sensibles que impedían el repatriación de los exiliados, como el cierre de las causas judiciales abiertas durante el período del terrorismo estatal y/o después de 1983, y la reversión de su estigmatización social y política ${ }^{4}$.

En este contexto poco favorable, quienes lograron entrar al país y comenzaron a insertarse nuevamente debieron enfrentar el fuerte cambio cultural, político y económico de la sociedad post-dictatorial y una extendida percepción social hostil hacia los exiliados, caracterizada por la estigmatización, el rechazo explícito o el silencio, que tenían su origen tanto las imágenes impulsadas por la propaganda militar contra las acciones colectivas de denuncia político-humanitaria de los exiliados en la esfera internacional ("la campaña antiargentina"), sintetizadas en las populares y extendidas frases "por algo será" (que los mataron, que los desaparecieron, que se fueron) y "algo habrán hecho", y una asociación maniquea y mecánica entre exiliados y guerrilleros y/o terroristas.

En este articulos me propongo analizar y construir una primera tipología de las experiencias de exiliados argentinos en Venezuela ante la posibilidad política de regresar a su país. El trabajo se apoya en 32 trayectorias individuales (19 casos de exiliados retornados y 13 que se quedaron en Venezuela) reconstruidas a partir de testimonios editados, biografías y entrevistas realizadas con la metodología de la historia oral.

\footnotetext{
${ }^{3}$ Para un análisis comparativo de las políticas de estatales de paises del Cono Sur frente al retorno de exiliados de la ultima ola de exilios véase Sznajder y Roniger (2013: 364-371)

${ }^{4}$ LASTRA, Soledad, JENSEN, Silvina. La inscripción del retorno em la transición democrática. Apuntes para un abordaje sobre la jdicialización Del exílio argentino.Ponencia presentada en VI Seminario Internacional Políticas de la Memoria: 30 años de democracia en Argentina. Logros y desafios, Centro Cultural de la Mmoria Haroldo Conti, Buenos Aires, 7, 8 y 9 de noviembre de 2013. V. p. 13.
} 


\section{Retornar...}

De un total de 19 trayectorias de exiliados retornados a la Argentina encontramos que sus regresos se efectuaron en un espacio de tiempo que va desde la posguerra de Malvinas hasta el año 2007 y que obedecieron a diferentes razones y motivos, dando lugar a diversas estrategias. Dividimos este conjunto de casos en cuatro subgrupos de acuerdo a las características comunes que han presentado en su proceso de retorno, destacando las razones, motivos y percepciones que estuvieron detrás de la decisión.

En primer subgrupo es el de los retornos que se concretaron antes del fin de la dictadura y que estuvieron marcados por un fuerte deseo y entusiasmo por volver al país del que fueron forzados a exiliarse. Este fue el caso, por ejemplo, de una pareja de académicos exiliados en Mérida desde 1975 que volvieron "a explorar" en medio de la guerra de Malvinas, a pesar de que habían logrado insertarse en buenas condiciones en la Universidad de los Andes (ULA). Sus razones para regresar obedecieron a una decisión familiar de retornar apenas existieran las condiciones políticas ("queríamos volver, ya desde el año 1981"), al hecho de que extrañaban a su entorno familiar y de amigos, además de que les preocupaba que sus hijos crecieran y se arraigaran en Venezuela. La estrategia implicó la instalación de la esposa e hijos en la Argentina y la permanencia del marido trabajando en Venezuela hasta fines de 1983. Aunque regresaron sin un empleo asegurado, exploraron algunas opciones, hasta que lograron ser reincorporados como docentes de la Universidad Nacional del Comahue cuando se inició el proceso de normalización de las universidades nacionales (Entrevista con Ricardo, La Plata, Argentina, 28/9/2010).

El segundo subgrupo esta integrado por retornos concretados entre 1983 y 1989 y agrupa a la mayoría de las trayectorias analizadas en este trabajo. Entre estos casos, ocho personas que retornaron a fines de 1983 aseveraron que decidieron volver al país ante el fin de la situación política que los había obligado a exiliarse - la existencia de condiciones políticas democráticas - y por la existencia de un fuerte deseo de retornar al país, a pesar de los problemas económicos, la continuidad del aparato represivo, la 
inestabilidad política y aunque la mayoría no tenía asegurada su inserción laboral cuando iniciaron el camino de regreso. A su vez, la mitad de ellos había salido al exilio con su familia y recordaron que la decisión de volver fue acordada con su pareja, destacando la preocupación por que los hijos no se arraiguen en Venezuela, cosa que evaluaban como un posible obstáculo para retornar si posponían la decisión por más tiempo (Entrevistas con Adolfo, Buenos Aires, Argentina 30/3/2009; Luisa, Buenos Aires, Argentina, 20/12/2008; Bernardo, Buenos Aires, Argentina ,15/11/2008; Carmelo, Ramos Mejía, Argentina , 6/03/ 2013).

Por otro lado, encontramos los casos de tres personas que retornaron en 1985. El primero es el de Munú, una ex militante de Montoneros liberada en julio de 1979 del campo de concentración que funcionó en la Escuela de Mecánica de la Armada. Luego de esperar un tiempo a que se aclare el panorama político de Argentina, y de solicitar a un abogado que interponga un habeas corpus por ella, Munú hizo un viaje exploratorio a mediados de 1984 bajo condiciones de estricta seguridad, entregó su testimonio en la Comisión Nacional sobre la Desaparición de Personas (CONADEP), y regresó a Caracas. A inicios de 1985 comenzó a pensar en la idea de retornar y decidió "probar que se siente volver a vivir en el país por dos meses" y para concretarlo realizó el tramite como refugiada del Alto Comisionado de las Nacionales Unidas para los Refugiados (ACNUR) para financiar su pasaje y una vez en Buenos Aires accedió a una media beca del Servicio Universitario Mundial (SUM) para terminar sus estudios en la Universidad Nacional de La Plata. Esta combinación de hechos la decidieron a quedarse en la Argentina, a pesar de la fragilidad de su condición económica y el temor a ser nuevamente víctima de desaparición, sensación que según recordó mantuvo durante toda la década de 1980 (Entrevista a Munú, Buenos Aires, Argentina, 6/11/2012). El segundo caso es el del sociólogo Julio Godio, exiliado en Maracaibo en 1975 por temor a ser víctima de los grupos parapoliciales que operaban en la ciudad de La Plata durante aquella época, y que se integró primero al Departamento de Economía de La Universidad del Zulia y luego, a partir de 1977, a la Fundación Friederich Ebert, desde donde ejerció cómo asesor laboral del gobierno del socialdemócrata Carlos 
Andrés Pérez (1974-1979). Godio afirmó que decidió regresar al país en 1983 apenas cambiaron las condiciones políticas que habían motivado su salida forzada y para ello contó con un contrato de trabajo de la Fundación Ebert. Sin embargo, la obtención de un puesto como funcionario de la Organización Internacional del Trabajo (OIT) sólo le permitió residir en el país a partir de 1985 (Entrevista a Julio Godio, Buenos Aires, Argentina, 30/3/2010). El tercer caso es el del académico platense José Sazbón que se exilió con su familia en Maracaibo en 1976, integrándose al Departamento de Economía de La Universidad del Zulia hasta su retorno a la Argentina en 1985, donde fue reincorporado como investigador titular del Consejo Nacional de Investigaciones Científicas y Técnicas (CONICET) y como docente universitario ${ }^{5}$. Luego encontramos el caso del ex sacerdote y ex detenido-desaparecido Patricio Rice y su pareja quienes retornaron en 1987. Según Rice la decisión de regresar estuvo relacionada con un conjunto de variables como: la no integración de su familia a Venezuela, el regreso de la mayoría de los exiliados argentinos que constituían sus relaciones sociales, el deseo de la pareja de regresar a la Argentina antes de que sus hijos sean más grandes, y su alejamiento del cargo de Secretario Ejecutivo de la Federación Latinoamericana de Familiares de Detenidos-Desaparecidos $(\text { FEDEFAM })^{6}$, un proyecto político-humanitario en el que había militado desde 1979. Al retornar vivieron dificultades laborales y económicas para su reinserción, decidiendo "comenzar desde cero" e instalándose en un barrio humilde de la localidad de Monte Grande en el sur del conurbano bonaerense. En aquel momento ninguno de los dos tenía profesión u oficio y no contaron con apoyo gubernamental para la reinserción (Entrevista a Patrick Rice, Buenos Aires, Argentina, 12/12/2008). Por último, exponemos el caso del retorno de Gabriel y familia en 1989. Ambos se habían exiliado en Venezuela en 1975 luego de que Gabriel fuera detenido y torturado por su militancia estudiantil secundaria y barrial en la zona de norte del conurbano

\footnotetext{
${ }^{5}$ TARCUS, Horacio. El último de los humanistas ardientes, Pagina 12, Buenos Aires, 7 de diciembre 2008.

${ }^{6}$ Analizo el rol de los exiliados argentinos en la constitución de la FEDEFAM en Ayala (2014).
} 
bonaerense. En 1983, ante la posibilidad del retorno, decidieron esperar un tiempo para observar como evolucionaba la situación política, aprovechando para trabajar y ahorrar para el retorno definitivo en el futuro. En 1988 realizaron un primer viaje exploratorio y decidieron retornar definitivamente en 1989 en una travesía familiar en camioneta a través de Brasil. Una vez en el país, tuvieron serios problemas para insertarse laboralmente en el contexto de crisis económica y política que vivía el país en ese entonces (Entrevista a Gabriel, Beccar, Argentina, 2/10/2010).

El tercer subgrupo esta compuesto por casos de retornos durante la década de 1990. Como el de la pareja de María y Ernesto, quienes volvieron en 1994, también en camioneta, en una decisión de retorno que fue posponiéndose por una mezcla de razones familiares, oportunidades laborales y profesionales en Venezuela. Habían huido del país en 1977 y se insertaron como profesionales en el Estado de Barinas, en los llanos venezolanos. $\mathrm{Su}$ reinserción laboral y la obtención de vivienda en Buenos Aires fueron recordados como difíciles, debiendo alquilar durante dos años, hasta lograr comprar un departamento con ahorros transferidos desde Venezuela. Ernesto consiguió trabajo como médico pediatra y María logró ser reincorporada como trabajadora estatal de la Provincia de Buenos Aires, luego de largas gestiones (Entrevista a María y Ernesto, Buenos Aires, Argentina, 19/12/2011).

En el cuarto subgrupo encontramos dos casos difíciles de caracterizar: uno de retorno en 2006 y otro de exploración para posible retorno de fines de 2007. Si bien ambos están fundados en motivos que se relacionan con su pasado-presente de haber sido forzados al exilio, también se encuentran cruzados por los problemas sociales y políticos de Venezuela en la última década, y por la percepción de una situación económica y política más estable en la Argentina, a la que valoran como positiva pues les permite acceder a una mejor calidad de vida. El primero es el caso de María Isabel quien afirmó que decidió retornar en 2006 luego de una evaluación muy profunda y de una "reconciliación con mi historia personal y con el país", aunque "con miedo a perder 
mi vida en Venezuela". Así, factores como el hecho de que sus hijos ya eran adultos, la formación de pareja con un ex exiliado retornado, la posibilidad de acceder a una mejor calidad de vida en Argentina además de valorar positivamente las políticas públicas del gobierno de Néstor Kirchner (2003-2007) — la decidieron a radicarse en Buenos Aires. Recordó que en 1983 no pensó retornar debido a que recién en esos años había logrado conseguir cierta estabilidad y condiciones de desarrollo personal (estudios de grado y comienzo de un posgrado), formado una nueva pareja, y porque no quería separar a sus hijos de su ex esposo, el cual tenía una causa judicial abierta que le impidió el retorno a la Argentina hasta avanzada la década de 1990 (Entrevista a María Isabel, Buenos Aires, Argentina, 9/10/2008).

El segundo caso es el de Viviana, asilada en la Embajada venezolana y deportada en 1979, que en diciembre de 2007 regresó a vivir un tiempo "para explorar" la posibilidad de radicarse en Argentina, o en un tercer país, pues la situación en Venezuela según su percepción se había tornado crítica en términos sociales, políticos y económicos y subrayó la fuerte inestabilidad y polarización política durante los dos primeros gobiernos de Hugo Chávez (1999-2000; 2000-2006). Desterrada cuando tenia veinte años de edad, volvió al país con cincuenta y ha realizado gestiones judiciales para lograr una indemnización como "víctima de exilio". Recordó haber viajado a Argentina el 31 de octubre 1983, con pasajes financiados por el ACNUR que le había otorgado el estatus de refugiada. Luego regresó en dos ocasiones en 1984 para explorar la posibilidad de retornar. Pero después de una estadía de seis meses en La Plata decidió volverse a Venezuela, puesto se le hacía insoportable el ambiente social, los recuerdos de la represión y el temor vivido, el cambio operado en la sociedad por el terrorismo estatal, y el estigma y sospecha que pendía sobre los exiliados. Después de 1984 se quedó en Venezuela y en la actualidad continúa explorando la posibilidad de quedarse (Entrevista a Viviana, City Bell, Argentina, 6/11/2008). 


\section{Cuedarse, permanecer, continuidad deexilio...}

Este apartado se apoya en 13 testimonios de exiliados que por diversas razones no retornaron, quedándose a vivir en Venezuela o que regresaron a la Argentina y luego emigraron a este país después de 1983. El grupo puede segmentarse al mismo tiempo en tres subgrupos: a) los que decidieron no retornar por libre elección; b) los que retornaron y luego de un tiempo decidieron regresar al país de refugio o emigraron a un tercer pais; y c) los que fueron forzados a posponer su retorno debido a procesos judiciales en su contra y que cuando los resolvieron decidieron radicarse de forma definitiva en Venezuela, y que plantean la cuestión de la continuidad de la política de exilio durante gobiernos democráticos?

El primer subgrupo esta compuesto por 7 casos que decidieron no retornar por libre elección consciente y a veces, en retrospectiva — según la reflexión de un entrevistado- inconsciente. Salvo dos entrevistados que afirmaron haber decidido no regresar desde el fin de la dictadura, los demás afirmaron haber transitado por diferentes actitudes y momentos, que fueron desde la idea de posponer la decisión de retorno hasta evaluar bien la situación política (en algunos casos realizando viajes exploratorios todos los años), hasta aquellos que vivieron tensiones internas entre regresar y no regresar por temor a la continuidad de la represión y también por cierto enojo o resentimiento con la sociedad argentina en tanto exclusora. Las trayectorias de Julio y Roque valen para ejemplificar los casos de quienes ya en 1983 habían decidido no retornar y continuar con su vida en Venezuela. En el caso de Julio, un abogado catamarqueño preso opcionado en 1975, para fines de la década del setenta había formado una familia nueva en Mérida y transformado rápidamente en un empresario exitoso. Afirmó no haber tenido la intención de retornar y que desde el inicio del exilio decidió que iba a vivir sin pensar permanentemente en volver, logrando

\footnotetext{
${ }^{7}$ Para un análisis de la continuidad de la política de exilio durante gobiernos democráticos en la región véase Sznajder y Roniger (2013).
} 
insertándose laboral y socialmente en Venezuela, aprovechando las oportunidades laborales y crediticias para formar empresas y hacer negocios (Entrevista a Julio, Mérida, Venezuela, 10/04/2011). Por su parte, Roque, un ex sacerdote tucumano que fue obligado a huir del país en 1975, había formado una familia en Venezuela y reformulado su vida y proyectos personales. En 1983 estaba esperando su tercer hijo y dedicándose al trabajo militante en la capacitación a organizaciones campesinas en la Península de Paria, estado Sucre, como miembro de Centro al Servicio de la Acción Popular - CESAP (Entrevista a R. Carmona, Los Teques, Venezuela, 03/04/2011). En ambos casos, el hecho de haber formado una familia con una pareja venezolana, los proyectos personales de trabajo y de militancia y una integración en la sociedad venezolana fueron motivos por los cuales no consideraron retornar y se transformaron en migrantes luego de 1983.

Los casos de aquellos exiliados que decidieron posponer la decisión de retorno hasta evaluar bien la situación política — pero también la económica, legal y represiva - y que fueron quedándose sin nunca haber tomado la decisión de quedarse, podemos ejemplificarlo con la experiencia de Francisco, un abogado laboralista que en los setenta militaba en el "peronismo de izquierda" en Rosario, y que debió exiliarse con su familia en 1975. Francisco primero estuvo tres años en Lima trabajando como asesor sindical y docente universitario hasta que el empeoramiento de la situación política en este país hizo que se trasladara con su familia a Venezuela con el apoyo de la Central Latinoamericana de los Trabajadores (CLAT) ${ }^{8}$. Una vez instalado en Caracas logró revalidar su título, se insertó como abogado laboralista, realizó un posgrado y ganó un concurso de profesor en la Universidad Central de Venezuela (UCV). Recordó que en 1983 se encontraba estabilizado en Venezuela y que su esposa no quería retornar, motivos por los cuales decidió posponer la idea de retornar a la Argentina. Sin embar-

\footnotetext{
${ }^{8}$ La CLAT fue creada el 8 de diciembre de 1954 en Santiago de Chile como sede latinoamericana de la Confederación Mundial del Trabajo (CTM), de orientación socialcristiana, e inspirada en la doctrina del humanismo integral. Desde sus inicios dio énfasis a formación de los cuadros sindicales y lo reforzó a partir de la creación de la Universidad de los Trabadores de América Latina (UTAL) en 1974 (Soneira, 2008:333). Ambas sedes se encontraba en las afueras de Caracas.
} 
go, desde mediados de 1982 realizó viajes exploratorios todos los años, hasta que comprobó que no existían condiciones políticas para desarrollar una militancia y actividad profesional como la que había realizado en los años setenta, ni como la que mantenía en ese momento en Venezuela con sindicatos y gremios, a lo cual se sumaba una percepción negativa de la impunidad de los crímenes de los militares con que convivía la sociedad argentina. Por lo tanto, el no retorno en su caso tuvo que ver, por un lado, con una estrategia familiar que se combinaba con una situación estabilidad laboral y posibilidades de crecimiento profesional y, por el otro, con sus desencuentros con la sociedad, la política, la impunidad de los crímenes de la dictadura y la conciencia de la derrota del proyecto político en el que había militado en los setenta (Entrevista con Francisco, Caracas, Venezuela, 8/4/2011).

Entre los que vivieron tensiones internas entre regresar y no regresar por temor a ser nuevamente victimas de la represión y que al mismo tiempo sintieron rechazo y enojo con la sociedad argentina de la época, podemos mencionar el caso de Roberto, un ingeniero catamarqueño, ex funcionario del gobierno camporista en su provincia, preso político entre 1975 y 1979, y que logró salir a Francia en abril de 1982. A fines de este año se radicó en Mérida, gracias al apoyo de otros exiliados argentinos en esa ciudad. Roberto recordó haber salido del país con una sensación contradictoria, mezcla de asombro y sorpresa, rechazo a la sociedad argentina y miedo de la represión. Había permanecido aislado cinco años en un pabellón de máxima seguridad de la cárcel de Sierra Chica y cuando fue liberado vivió en carne propia los efectos de la dictadura en la sociedad y las movilizaciones de apoyo a la invasión militar de las Islas Malvinas. También recordó haber quedado marcado de forma traumática por la destrucción de sus escritos al momento de salida de la cárcel, fruto de casi cinco años trabajo, entre los cuales se encontraba una novela. A fines de 1983 recién había comenzado a reconstruirse a nivel personal y laboral. Atravesado por un deseo de retornar combinado con un rechazo al país y a los cambios sociales operados por los efectos del terrorismo de Estado, fue posponiendo la decisión hasta que perdió todas las expectativas 
y el entusiasmo que le habían generado algunas medidas y promesas de gobierno de Raúl Alfonsín -al que consideró "el mayor traidor"- . Afirmó además que decidió quedarse en Venezuela luego de la aprobación de las Leyes de Punto Final (1986) y Obediencia Debida (1987) que consagraban la impunidad de los crímenes de la última dictadura cívico-militar. Para esa época ya había comenzado una nueva relación de pareja con la que habían tenido dos hijos.

El segundo subgrupo es el de aquellos que retornaron a mediados de la década del ochenta y que luego de un tiempo decidieron volver a continuar su vida en Venezuela. El primer caso es el de Ricardo Mitre, un director de teatro porteño exiliado en Caracas en 1973 debido a amenazas de la Triple A. En 1985 decidieron retornar en familia, pues consideraban que las condiciones políticas que habían motivado su exilio ya no existían. Sin embargo, luego de vivir tres años en Buenos Aires decidieron radicarse nuevamente en Venezuela. En su testimonio recordó que vivieron fuertes desencuentros con la sociedad posdictatorial y que experimentaron el rechazo y el estigma social generalizado contra los exiliados. Llegando incluso a buscar agruparse con otros ex exiliados en Venezuela, autoidentificándose grupalmente como "argenven". También destacó que el retorno lo vivieron como un nuevo exilio y que esta situación les generó contradicciones y tensiones, hasta que decidieron que "su lugar en el mundo" estaba en Venezuela. Cuando en 1988 llegaron a Caracas tuvieron la "sensación de volver a casa" (Testimonio de Ricardo Mitre, en Martínez Ubieda, 2006). El segundo caso es el de José, un tucumano preso opcionado en 1975, que retornó en 1985 con la intención de reinsertarse política y laboralmente y que decidió regresar a Venezuela en 1988 debido a una combinación de factores que fueron desde el desencuentro con los cambios sociopolíticos de Argentina durante la dictadura, el rechazo y el estigma hacia los exiliados, la crisis económica, la imposibilidad de continuar una militancia como la que había practicado en los años sesenta y setenta, y el descubrimiento de una causa judicial abierta en su contra en un Juzgado de la Provincia de Tucumán. 
El tercer subgrupo de exiliados no retornados es del de aquellos que fueron forzados a posponer su retorno debido a la existencia de procesos judiciales en su contra y que para cuando fueron resueltos en la década del noventa, ya habían tomado la decisión de radicarse de forma definitiva en Venezuela. El primer ejemplo el caso de Raúl, militante de Montoneros sobreviviente del campo de concentración de la ESMA que se exilió en Venezuela en 1979. Raúl y su compañera, Rosario, sobreviviente del mismo campo de concentración, fueron activos denunciantes de las violaciones de los derechos humanos de la dictadura en los foros internacionales. En los inicios de la transición democrática presentaron su testimonio en la CONADEP y en 1985 viajaron a la Argentina con pasajes financiados por el ACNUR con la intención de explorar el contexto e intentar el retorno en 1986. La pareja recordó que durante esta época se habían reconstruido en Venezuela, que temían ser víctimas de la represión nuevamente y que durante esos años los abogados defensores de los militares le iniciaron a Raúl un juicio por falso testimonio, que terminó en la apertura de una causa en su contra que le impidió su ingreso al país hasta avanzada la década de 1990. Cuando esta causa prescribió y estaban en condiciones de retornar decidieron familiarmente que ya era tarde para desarraigar a sus hijos adolescentes y pequeños del país que los había acogido, y además, ellos se habían integrado fuertemente a la vida social y política venezolana. El segundo caso es el de Raúl-2 quien ante la apertura democrática en la Argentina decidió posponer la idea de retorno debido a proyectos profesionales, laborales, y a la formación de una nueva pareja con una venezolana, situación que se completó con el descubrimiento de la existencia de una causa judicial en su contra que le impedía regresar a la Argentina y que prescribió recién en la década del noventa. Como resultado de esta situación decidió radicarse definitivamente en Venezuela e incluso obtuvo la ciudadanía venezolana.

\section{observaciones Finales}

La etapa final de la última dictadura militar y el inicio de la larga transición a la democracia en la Argentina a partir de fines de 1983 puso 
a los exiliados argentinos en Venezuela ante una serie de evaluaciones y decisiones políticas, personales y grupales complejas donde el retorno fue una posibilidad entre otras. El análisis del grupo de casos analizados en este articulo muestra que la mayoría de los retornos de exiliados argentinos en Venezuela se realizaron entre los años 1983 y 1985, y que luego se espaciaron en un proceso que llega hasta el presente.

La decisión del retorno tuvo una intima relación con el deseo de conjurar el desarraigo provocado por la salida forzada y con la idea de retomar la vida familiar, social y política en el país de origen. En cambio, las estrategias para concretarlo fueron diversas: desde el impulso inicial de regresar apenas cambiaran las condiciones políticas mínimas, hasta esperar a reunir ahorros, terminar estudios, o esperar una estabilización de la situación política y el fin de la política represiva.

Los retornos se concretaron en un contexto muy poco favorable para la reinserción caracterizado por la debilidad del gobierno democrático, la recesión y crisis económicas, la existencia de un extendido estigma social hacia los exiliados, la posibilidad de ser represaliado nuevamente. Además, el regreso al país se tornó aún más difícil por no existir una política estatal coherente y activa de apoyo a la reinserción de los exiliados retornados por parte del gobierno nacional, como lo demuestra el contraste con los casos de Uruguay o Chile9.

Pero también hubo muchos exiliados que en 1983 decidieron no retornar, otros que simplemente se fueron quedando y un grupo que fue forzado a permanecer fuera del país debido a procesos judiciales en su contra iniciados durante el periodo de terrorismo estatal y también en la década del ochenta, los cuales prescribieron recién avanzada la década de 1990, lo cual plantea el tema de la continuidad del exilio bajo regímenes democráticos.

La tipología esbozada en este trabajo coincide con las "posibles rutas de postexilio" que elaboraron Sznajder y Roniger en su estudio sobre la

\footnotetext{
${ }^{9}$ Para un análisis comparativo de las políticas de estatales de paises del Cono Sur frente al retorno de exiliados de la ultima ola de exilios véase Sznajder y Roniger (2013: 364-371)
} 
políticas de destierro y exilio en América Latina: 1) Expatriación e integración en el extranjero; 2) retorno; 3 ) volver la país de origen y emigrar (al país de exilio o a un tercer país), 4) permanecer en condiciones de exilio; 5) muerte y repatriación ${ }^{10}$. Además, también coincidiendo con estos autores, encontramos que los caminos del postexilio son múltiples y que volver es solo una de las opciones.

Todos los entrevistados destacaron que en la decisión del volver o quedarse hubo una preocupación por el destino de sus hijos. A los que retornaron les preocupaba que sus hijos no crecieran y se arraigaran en Venezuela porque lo evaluaban como un posible obstáculo para repatriarse si posponían la decisión por más tiempo.

En contraste con otras experiencias de retornos organizados políticamente como el de los militantes de partidos uruguayos o chilenos de la última ola de exilios, la mayoría los casos de argentinos analizados en este trabajo afirmaron que la decisión de retornar, permanecer o re-emigrar, fue decidida mayormente en el ámbito personal-familiar. Sin embargo la dimensión política de la decisiones en el postexilio fue significativa: si bien la mayoría de los exiliados habían pertenecido a organizaciones políticas que fueron derrotadas y aniquiladas en los primeros años del exilio, varios de ellos una vez en el exterior se sumaron y/o construyeron proyectos políticos centrados en la denuncia humanitaria y la búsqueda de la solidaridad internacional en los piases de acogida, mientras que otros se integraron a organizaciones políticas y sociales venezolanas.

${ }^{10}$ SZNAJDER, Mario, RONIGER, Luis. La política del destierro y el exílio en América Latina. México: Fondo de Cultura Económica, 2013, p. 371. 